
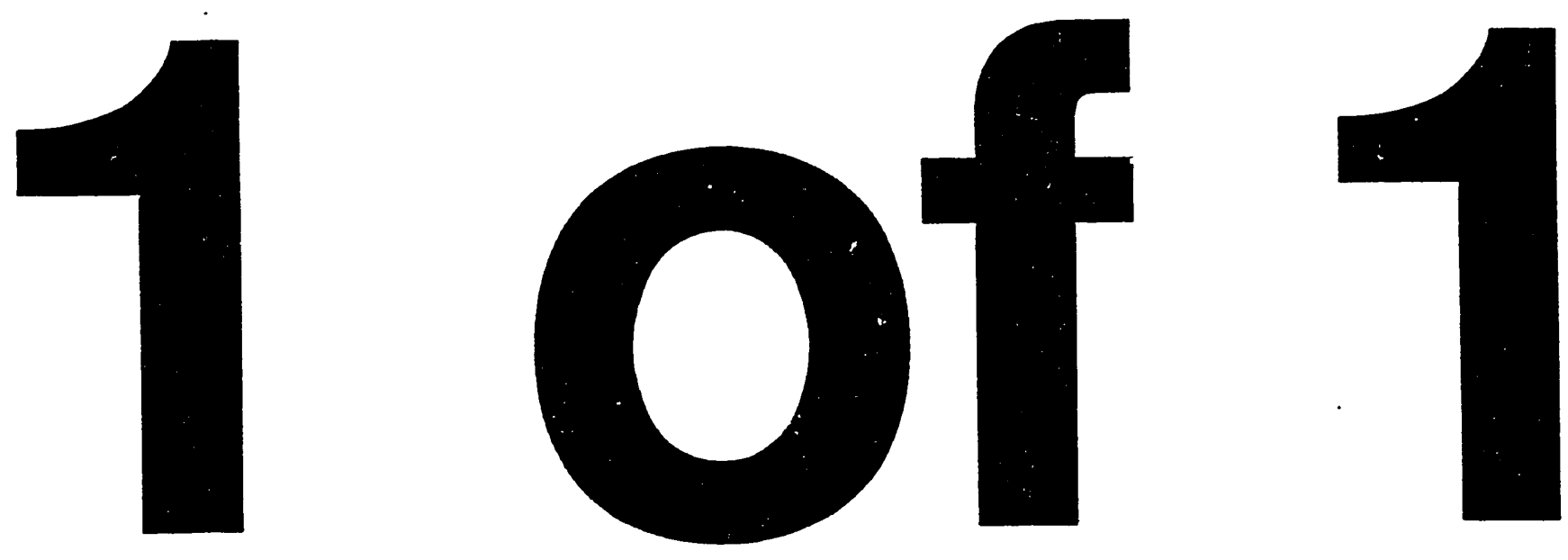
LBL-35002

\title{
ESTIMATING THE COST OF LARGE SUPERCONDUCTING THIN SOLENOID MAGNETS
}

\author{
M. A. Green \\ Lawrence Berkeley Laboratory \\ University of California \\ Berkeley, CA 94720 \\ S. J. St. Lorant \\ Stanford Linear Accelerator Center \\ Stanford University \\ Stanford CA. 94309 \\ Cryogenic Engineering Conference \\ Albuquerque Conference Center \\ Albuquerque, New Mexico \\ 12-16 July 1993 \\ To be published in the \\ Advances in Cryogenic Erigineering Vol 39
}

*This work was performed at the Lawrence Berkeley Laboratory with the support of the Director of the Office of Energy Research, Office of High Energy and Nuclear Physics, High Energy

Physics Division, U. S. Department of Energy under Contract No. DE-AC03-76SF00098. 


\title{
ESTIMATING THE COST OF LARGE SUPERCONDUCTING THIN
}

\section{SOLENOID MAGNETS}

\author{
M. A. Green \\ Lawrence Berkeley Laboratory \\ University of $\mathrm{Ca}^{-}$ifornia \\ Berkeley, CA 94720
}

\section{S. J. St. Lorant}

Stanford Linear Accelerator Center

Stanford University

Stanford, CA 94309

\begin{abstract}
The cost of thin superconducting solenoid magnets can be estimated if one knows the magnet stored energy, the magnetic field volume product or the overall mass of the superconducting coil and its cryostat. This report shows cost data collected since 1979 for large superconducting solenoid magnets used in high energy physics. These magnets are characterized in most cases by the use of indirect two phase helium cooling and a superconductor stabilizer of very pure aluminum. This correlation can be used for making a preliminary cost estimate of proposed one of a kind superconducting magnets. The magnet costs quoted include the power supply and quench protection system but the cost of the helium refrigerator and helium distribution system is not included in the estimated cost.
\end{abstract}

\section{INTRODUCTION}

It is often difficult to get a budgetary estimate of the cost of a detector type of superconducting magnet system and the helium refrigeration system needed to keep it cold. This report presents a method for making a budgetary cost estimate of relatively light weight superconducting solenoid magnets based on the known costs of similar types of magnets. One of the difficulties with this kind of estimate is the choice of the appropriate scaling parameter. In some cases it makes little difference which parameter is chosen, but in other cases, the cost estimates are wildly different depending on the parameter chosen. As a class superconducting magnets of the so called "thin detector solenoid" (a magnet with minimum radiation thickness for particles to pass through) can exhibit very different behavior depending on the scaling parameter chosen. The reasons for widely varying estimated cost as a function of scaling parameter for this class of magnets is dealt with in this report. 


\section{THE COST OF DETECTOR TYPE SUPERCONDUCTING MAGNETS}

As superconducting magnet systems increase in size and complexity, it is appropriate to analyze the corresponding tre $d \mathrm{~s}$ in the costs of the major components: the magnets themselves and the refrigeration required to maintain them in operation. Every so often, such an analysis appears in print, usually directed at specific applications. In the early seventies, wizen advances in plasma physics made prototype fusion reactors feasible, a number of interesting economic assessments of such devices were published:1,2 Ten years later, superconducting energy storage reached respectability and so its economics were scrutinized. 3,4 The purpose of this paper is to take a representative cross-section of thin superconducting solenoid systems anci using known costs, fit known cost equations for these types of superconducting magnets onto a 1993 cost basis.

The composition of our sample includes eleven large relatively thin detector magnets with iron return paths (most of the world's large particle physics detector magnets); one magnet is a system of three large solenoids which forms a dipole magnet ing with the flux returned by a ring of $C$ shaped iron; and one magnet is a bath cooled solenoid without an iron return path which is used a detector magnet. Ten of the magnets are wound with superconductor stabilized with pure aluminum matrix and the other three were wound with a copper matrix superconductor. In size, the magnets varied from a 1.2 meter bore solenoid magnet to a system of large solenoids, the largest of which is 15.1 meters in diameter. The useful central magnetic induction for the magnets varies from 0.7 to $2.0 \mathrm{~T}$. The range of stored magnetic energies when the magnets run at their at the design central induction is from 3.4 to $130 \mathrm{MJ}$. The field times useful field volume for the magnets included in this report varied from 5.1 to $250 \mathrm{~T} \mathrm{~m}^{3}$. The mass of the finished magnets and cryotats varied from 3.6 to 84 metric tons $(\mathrm{Mg})$. Only fabricated magnet systems, where the cost of manufacture is known, were included in the survey. Magnet studies, planned projects and the like were excluded. The magnets included in the survey were manufactured in countries from Europe, Asia and North America. Over half of the magnets were fabricated by industrial companies; the remainder were fabricated by various national laboratories.

\section{Methodology}

The system characteristics were obtained from a systematic perusal of the published literature, which included technical reports circulated among interested institutions, and confirmed by direct inquiry. For the costs, the "Technical Proposal" or its equivalent was the usual starting point, followed by an actual tracking of the project costs through information obtained from the funding agency or its representative organ. In the US, this is often simply a matter of identifying the appropriate government publication; abroad, it requires a network of helpful correspondents and friendly reciprocity. In spite of the disparity of the sources, the raw cost data were usually reliable to about 15 or 20 percent.

In most cases, the magnet system was assumed to be compieted on the date of its first successful acceptance test. The purpose of this artificial cut-off is to better isolate the construction costs from subsequent tuning improvements which tend to have a life of their own and hence associated costs of their own. The actual project cost was then converted to 1993 dollars using the composite escalation index for large construction projects. Foreign project costs were converted to US currency using the exchange rate at the time of construction and then they were escalated in the same manner as domestic projects.

Three parameters were used to characterize each system: the energy stored in the magnetic field, the field-magnetic volume product in the volume between the iron poles, and the total mass of the coil and cryostat without the cryogen. Stored energy is a useful parameter when coil systems are strain limited. The field times volume product defines the extent of thie magnetic field. The mass of the magnet coil and cryostat is in certain instances a better measure of the magnet system cost than either stored energy or the product of magnetic field and volume because it defines the cost as a function of the amount of material used to fabricate the magnet system. 


\section{Results}

Figures 1,2 and 3 are the scatter diagrams of the cost-magnet parameter relationships for the entire sample. The lines in each figure are linear least square fits to the data points in log-log space. The overall cost of the magnets given in Fig. 1 can be represented by the following equation:

$$
C(M \$)=0.458[E(M)]^{0.700}
$$

Where the cost C is given in millions of 1993 US dollars and the stored magnetic energy $\mathrm{E}$ at the magnet design current is given in MJ. The correlation parameter between Equation 1 and the data (on a log-log plot) is 0.74

The overall cost of the magnets given in Figure 2 can be represented by the following expression:

$$
C(M \$)=0.376\left[G\left(\mathrm{Tm}^{3}\right)\right]^{0.655}
$$

where $C$ is the magnet cost given in millions of 1993 US dollars and G is the fieldmagnetic volume product when the magnet is operating at its design curre it given in tesla times meter cubed. The correlation parameter between equation 2 and the data is 0.77 .

The overall cost of the magnets given in Figure 3 can be characterized by the following expression:

$$
C(M \$)=0.559[M(\text { tons })]^{0.719}
$$

where $C$ is the magnet cost given in millions of 1993 US dollars and M is the overall mass of the coil and cryostat given in metric tons $(\mathrm{Mg})$. The correlation parameter between the data and Equation 3 is 0.65 .

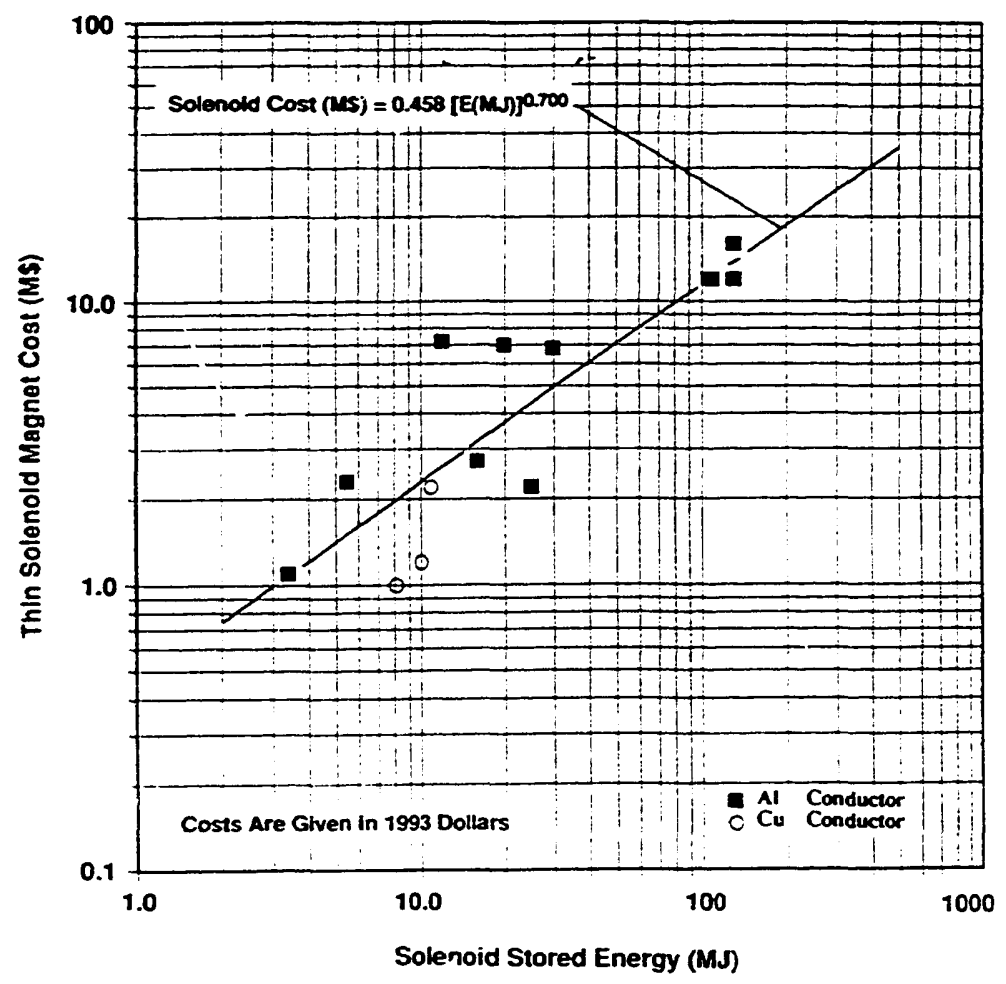

Figure 1. Superconducting Magnet Costs Versus Magnet Stored Energy. 


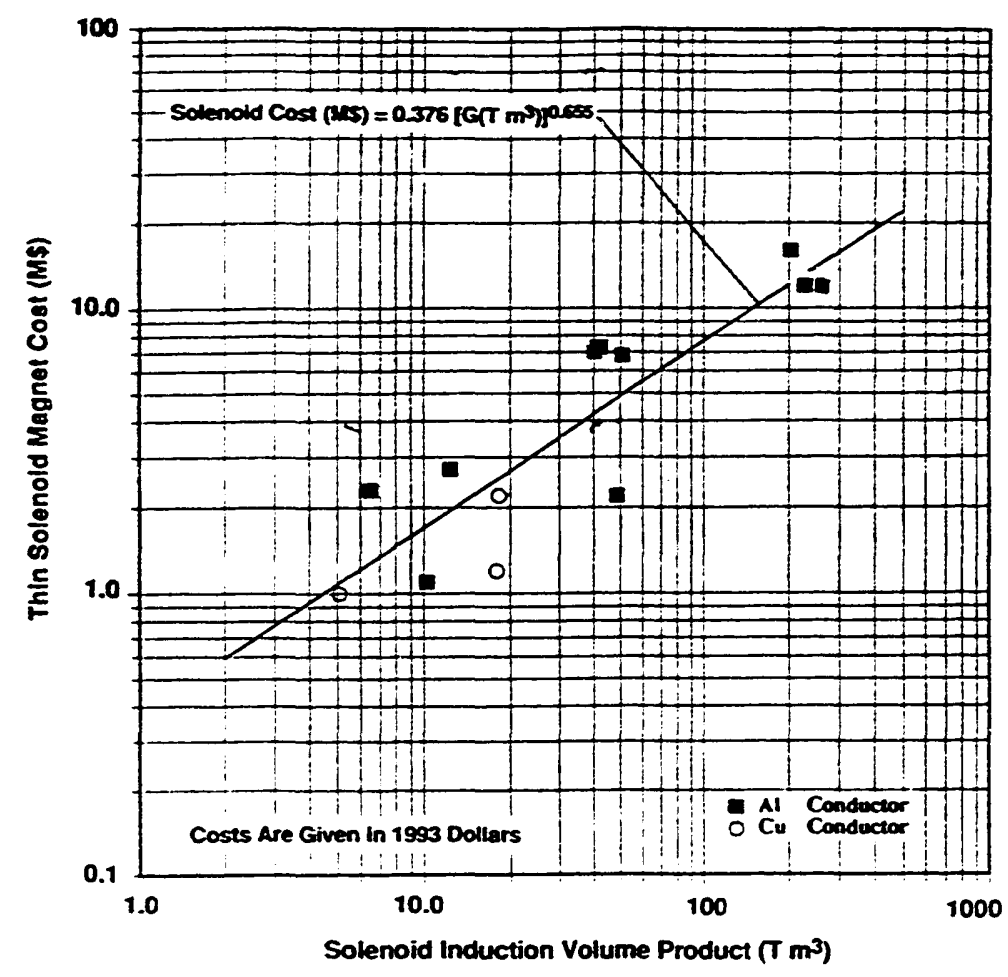

Figure 2. Superconducting Magnet Costs Versus Field-Magnetic Volume Product.

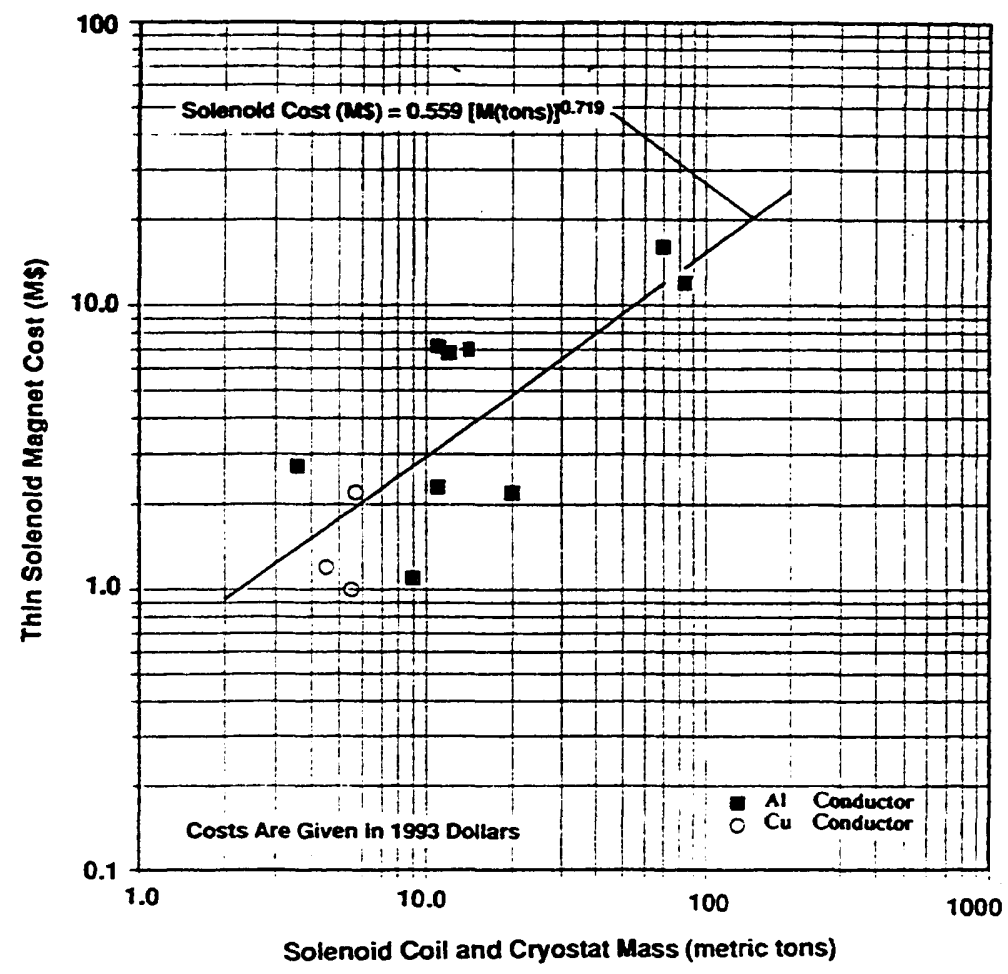

Figure 3. Superconducting Magnet Cost Versus Overall Coil and Cryostat Mass. 


\section{DISCUSSION}

It is interesting to compare cost equations 1 and 2 with the cost equations for solenoids and split solenoids developed for reference 5 in 1991. The cost equations given in reference 5 were multiplied by 1.08 to reflect inflation between the summer of 1991 and the summer of 1993. For general solenoid type of magnets, the cost equations for 1993 take the following form:

$$
C(M \$)=0.565[E(M)]^{0.662}
$$

and

$$
C(M \$)=0.937\left[G\left(\mathrm{Tm}^{3}\right)\right]^{0.577}
$$

where $\mathrm{C}$ is the cost given in millions of 1993 US dollars; $\mathrm{E}$ is the magnet design stored energy given in M!; and $G$ is the central induction time volume given in tesla times meter cubed. It is interesting to note that the slope of the cost curves is lower for solenoids in general than it is for the thin solenoids.

If the data from one area of the world (where costs are high) is excluded from the data which was fit, Equations 1 through 3 take a different form. The curve fits done on the basis of the ten magnets from less expensive parts of the world take the following form:

$$
\mathrm{C}(\mathrm{M} \$)=0.351[\mathrm{E}(\mathrm{M})]^{0.727}
$$

and

$$
C(M \$)=0.357\left[G\left(\mathrm{Tm}^{3}\right)\right]^{0.635}
$$

and

$$
\mathrm{C}(\mathrm{M} \$)=0.422[\mathrm{M} \text { (tons) }]^{0.747}
$$

where $C, E, G$, and $M$ are defined as previously defined. The correlation parameters for the data with Equations 1a through $3 \mathrm{a}$ (on a $\log -\log$ plot) are $0.88,0.83$ and 0.79 respectively. If one is going to estimate the cost of thin solenoid, when one can buy the magnet at the lowest price on the world market, Equations la through 3a are better to use than Equations 1 through 3

When doing a cost estimate of a magnet which has construction technique similar to the large detector magnets, one should use all three of the cost equations. Problems occur when the costs calculated using the three equations don't agree (to say 20 or 25 percent). It takes a certain amount of judgement to estimate the magnet cost under these circumstances.

As an example, the data points used to generate the cost versus stored energy equation were for the most part dominated by cases where the magnet structure was magnetic stress limited. If one has a very large low-field magnet, the stored energy will be low even though the field volume is large. This type of magnet will not be magnetic stress limited. Instead, the magnet is dominated by the amount of material needed to support the structure against gravity and vacuum loading. The use of Equation 1 or Equation la will generate a cost estimate which is low. In the case of a large-volume low-field magnet, where the coil is not magnetic stress limited, it is appropriate to use Equations 2, 2a, 3 or 3a for estimating cost.

Twelve of the thirteen magnets with data points in Figures 1 through 3 are simple solenoids with all coils hooked up in series so that the fields are additive. The thirteenth is a system of three large solenoids where two solenoids are hooked in opposition to the third 
solenoid. As a result, most of the stored energy and magnetic field is between the solenoids. Both stored energy and magnetic field times volume are restricted by such an arrangement. In a case such as this, it is better to use Equations 3 or $3 a$ to estimate cost.

Other factors which influence the cost of a thin detector solenoid include the following: 1) The techniques used to make the vacuum vessel thin (light weight) will greatly affect cost. As an example, large magnets which have honey comb or composite structure vacuum vessels will be more costly than magnets made with simple aluminum cylindrical shell vacuum vessels. 2) The number of pieces of coils which are wound have an effect on cost. A long thin solenoid which is wound as a single coil will be more expensive than a long thin solenoid which consists of a number of coils which are assembled to form a single coil. Subdivision of the crycstat does not, as a rule, achieve similar cost savings. 3) Beyond a certain size, the magnet has to be wound and assembled on site. Most of the magnets which,were are included in the data sets in Figures 1 through 3 were wound and assembled at one site and shipped to another site. Transport of the coil was a real problem for some the magnets included in the data set. One magnet system included in the data set consists of two coils which are 13.4 meters in diameter and one coil which is 15.1 meters in diameter. This magnet system was manufactured and assembled on site. 4) The cost of the superconductor is a factor in estimating the cost of the magnet. Most of the thin detector type magnets which are in the data set have been made from very pure aluminum stabilized superconductor. The cost of this conductor varies widely (from $\$ 75$ to $\$ 575$ per kilogram) depending on the vendor. A magnet design where a significant portion of the magnet and cryostat mass is conductor will be affected by the choice of the superconductor vendor.

\section{ACKNOWLEDGMENTS}

This work was performed at the Lawrence Berkeley Laboratory with the support of the Director, Office of Energy Research, Office of High Energy and Nuclear Physics, High Energy Physics Division, U. S. Department of Energy under Contract No. DE-AC0376 SF00098.

\section{REFERENCES}

1. M. S. Lubell, H. M. Long, J. N. Luton, and W. C. T. Stoddart, Applied Superconductivity Conference Proceedings, Annapolis, MD, p 341, (1972).

2. J. R. Powell, Applied Superconductivity Conference Proceedings, Annapolis, MD p 346, (1972).

3. B. M. Winer and J. Nicol, IEEE Transactions on Magnetics MAG-17, No. 1, p 336 (1981).

4. R. W. Boom, IEEE Transactions on Magnetics MAG-17, No. 1, p 340 (1981).

5. M. A. Green, R. A. Byrns and S. J. St Lorant, Advances in Cryogenic Engineering 37a, p 637, Plenum Press, New York (1991) 


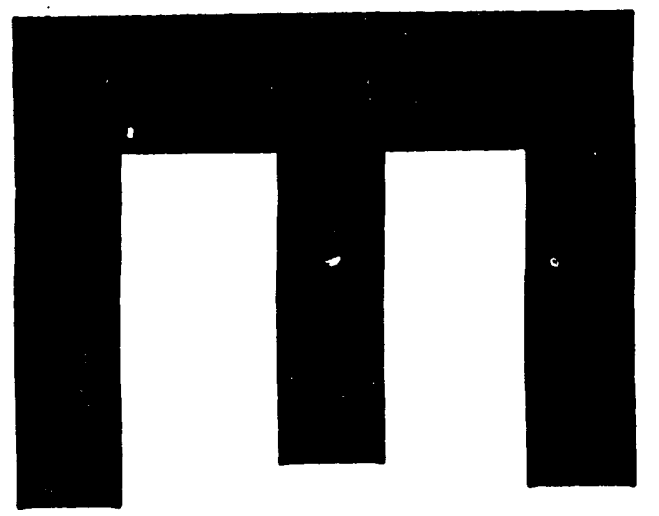

$\omega$
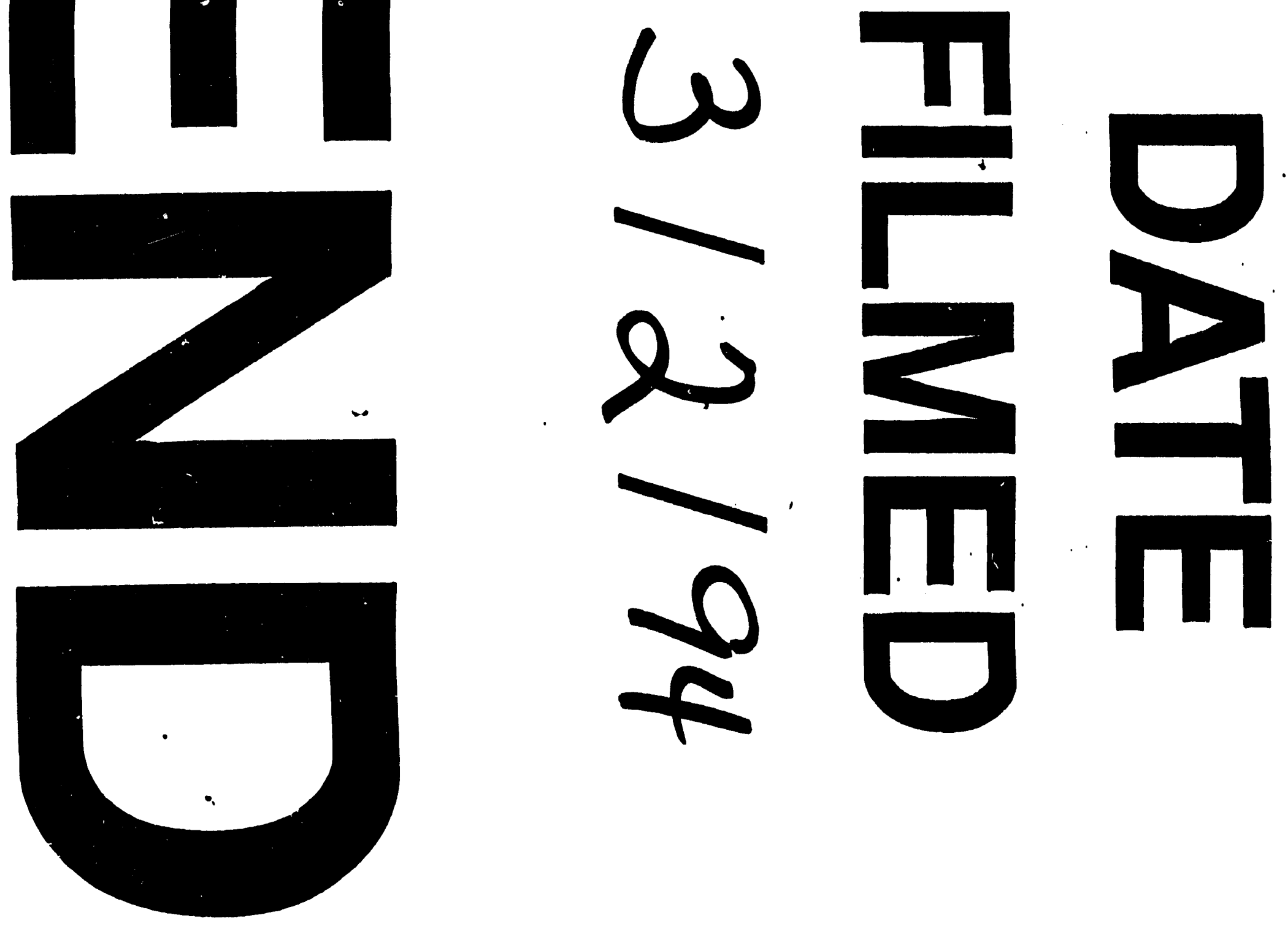